\title{
Construction of an integrative regulatory element and variation map of the murine Tst locus
}

\author{
Jasmina Beltram', Nicholas M. Morton², Tanja Kunej ${ }^{1}$ and Simon Horvat ${ }^{1,3^{*}}$
}

\begin{abstract}
Background: Given the abundance of new genomic projects and gene annotations, researchers trying to pinpoint causal genetic variants are faced with a challenging task of how to efficiently integrate all current genomic information. The objective of the study was to develop an approach to integrate various genomic annotations for a recently positionally-cloned Tst gene (Thiosulfate Sulfur Transferase, synonym Rhodanese) responsible for the Fob3b2 QTL effect on leanness and improved metabolic parameters. The second aim was to identify and prioritize Tst genetic variants that may be causal for the phenotypic effects.

Results: A bioinformatics approach was developed to integrate existing knowledge of regulatory elements of the Tst gene. The entire Tst locus along with flanking segments was sequenced between our unique polygenic mouse Fat and Lean strains that were generated by divergent selection on adiposity for over 60 generations. The bioinformatics-generated regulatory element map of the Tst locus was then combined with genetic variants between the Fat and Lean mice and with comparative analyses of polymorphisms across 17 mouse strains in order to prioritise likely causal polymorphisms. Two candidate regulatory variants were identified, one overlapping an evolutionary constrained Tst intronic element and the other residing in the seed region of a predicted $3^{\prime} U T R$ miRNA binding site.
\end{abstract}

Conclusions: This study developed a map of regulatory elements for the Tst locus in mice and identified candidate genetic variants with increased causal likelihood. This map provides a basis for experimental validation and functional analyses of this novel candidate leanness and antidiabetic gene. Our methodological approach is of general utility for analyzing regulation of loci that have limited annotations and experimental evidence and for identifying candidate causal regulatory genetic variants in post-GWAS or post-QTL- cloning studies.

Keywords: Thiosulfate sulfurtransferase (TST), Rhodanese, Regulatory elements, Map, Obesity, Lean, Gene variants, Polymorphism, Bioinformatics

\section{Background}

In the last decade we have witnessed intense efforts to identify genetic variants controlling complex traits in various species. In humans, for example, genome wide association studies (GWAS) have identified several thousand gene variants associated with complex traits and diseases [1]. These gene variants can include structural variants such as insertion/deletions, in-frame deletions

\footnotetext{
* Correspondence: simon.horvat@bf.uni-lj.si

${ }^{1}$ Biotechnical Faculty, Animal Science Department, University of Ljubljana, Groblje 3, 1230 Domzale, Slovenia

${ }^{3}$ National Institute of Chemistry, Hajdrihova 19, 1000 Ljubljana, Slovenia Full list of author information is available at the end of the article
}

(indels), inversions, copy-number variants (CNVs) and most frequently (over $95 \%$ ) single nucleotide polymorphism (SNP) markers [2, 3]. A majority of variants mapped to candidate regions are unlikely to be causal for the effect on the trait [4]. They likely represent closely linked genetic markers that are in linkage disequilibrium (LD) with the causal variant. In animal models loci for complex traits (quantitative trait loci; QTL), can also be detected using GWAS approaches, especially in outbred species. However, in inbred laboratory animal models complex trait loci are more frequently identified using genetic analyses of crosses between strains differing in the trait(s) of interest. 
Animal models offer additional complementary methods for identifying candidate genes and their related pathways in humans. Advantages of using animal genetic models are the ability to better control environmental factors, genetic models are less heterogeneous, and gene expression can be examined in some tissues not readily available from humans. Finally, genes found for complex traits in animal models are often conserved in humans (e.g., [5-7]).

A major hurdle in GWAS or QTL studies is to proceed efficiently from detected markers linked to the candidate loci of interest to identification of the causal genetic variant responsible for the phenotypic effect. This holds true also for cases where the locus responsible for a QTL effect has been identified (e.g. by a combination of genetic mapping, expression and transgenic studies) but the genetic variant responsible within the locus has not been identified. Until recently, efficient prioritisation of candidate genetic variants based on bioinformatics analyses was hindered by limited functional annotation of the genome, especially outside coding sequences. However, in the recent years, several large-scale genomics projects such as Encyclopedia of DNA Elements (ENCODE; [8]), and the Functional Annotation of the Mammalian Genome (FANTOM; [9]), as well as improved genomic prediction tools now provide a comprehensive functional annotation in various cell and tissue types as well as developmental stages [8, 10-13]. Major advances in functional annotations are found in novel transcripts, promoters, enhancers, insulators, chromatin modification sites, transcription factor binding sites, as well as inter-species and intra-species conserved DNA elements.

Given the flood of new genomic projects and annotations, researchers looking for the best candidate causal genetic variants are faced with a challenging task of how to efficiently integrate all current genomic information. Although some genomic databases such as Ensembl [14] combine information from several different genome annotation projects, they still lack some information and tools to explore in detail a particular segment of the genome.

Our unique polygenic Lean (L) and Fat (F) mouse models have been described in detail in previous studies [15-17]. Briefly, original F and L lines were generated by divergent selection on adiposity for over 60 generations and at the end differed in body fat percentage by more than five-fold (Fat line, $23 \%$ body fat, Lean line, $4 \%$ body fat) $[15,16,18]$. Genetic studies have shown that the two polygenic mouse lines represent an excellent model for identifying genetic factors underlying the complex human obesity and leanness mechanisms. Several major quantitative trait loci (QTL) underlying fat divergence have already been described $[11,19,20]$ and some QTLs have been further mapped to a higher resolution and eventually positionally cloned [21, 22]. In the present study we focus on the nuclear-encoded mitochondrial thiosulfate sulfur-transferase (Tst, also known by synonym Rhodanese) that we recently identified in a positional cloning experiment as a causal gene for the Fob3b2 QTL phenotypic effect [22]. However, the genetic variants responsible for upregulation of Tst expression in the Lean mice have not yet been identified.

The main objective of the present study was to integrate various genomic annotations in the Tst locus to construct a map of regulatory elements of this gene and to identify and prioritise the causal genetic variants between the Fat and Lean lines. We focused on regulatory elements because the main driver of the phenotypic effect on leanness and metabolic parameters was an increase in expression of Tst in Lean compared to Fat mice [22]. To first uncover polymorphisms that may be causal for the difference in expression of the Tst gene, we undertook a classical high resolution Sanger-sequencing of the entire Tst locus in Fat and Lean lines. The identified genetic variants were then evaluated and prioritised using our regulatory element map of the Tst locus that integrated broad functional information from conserved polymorphisms in other strains, association studies, transcription factor binding site motifs, chromatin modification motifs and miRNA binding sites. Our approach to build a detailed integrative regulatory element map is of general utility as it can be applied for evaluating and prioritising polymorphisms within candidate regions in any trait or species of interest. Reducing and prioritising the number of potential causal polymorphisms is essential for efficient planning of further experiments to prove or support causality of candidate genetic variants. This may ultimately answer important basic research questions as well as provide a foundation for potential therapeutic developments.

\section{Methods}

Bioinformatics analysis -criteria for defining high priority regulatory sites

Bioinformatics analysis was performed on a $7400 \mathrm{bp}$-long segment of the mouse Tst gene, including the $\sim 0.5 \mathrm{~kb}$ upstream and downstream regions (Fig. 1; Additional file 1: Table S1, Additional file 2: Table S2, Additional file 3: Table S3, Additional file 4: Table S4, Additional file 5: Table S5, Additional file 6: Table S6, Additional file 7: Table S7, Additional file 8: Table S8, Additional file 9: Table S9, Additional file 10: Table S10). To define the high priority regulatory segments of the Tst gene we applied the following criteria - a) presence of at least one evolutionary constrained element b) a minimum of two other regulatory features such as open chromatin, transcription factor binding site, histone modification, RNA polymerase binding site, DNA methylation, $\mathrm{CpG}$ island or microRNA (miRNA) binding sites. These regulatory features were identified and mapped to the Tst locus using the bioinformatics databases with their tools or programs as described below. 


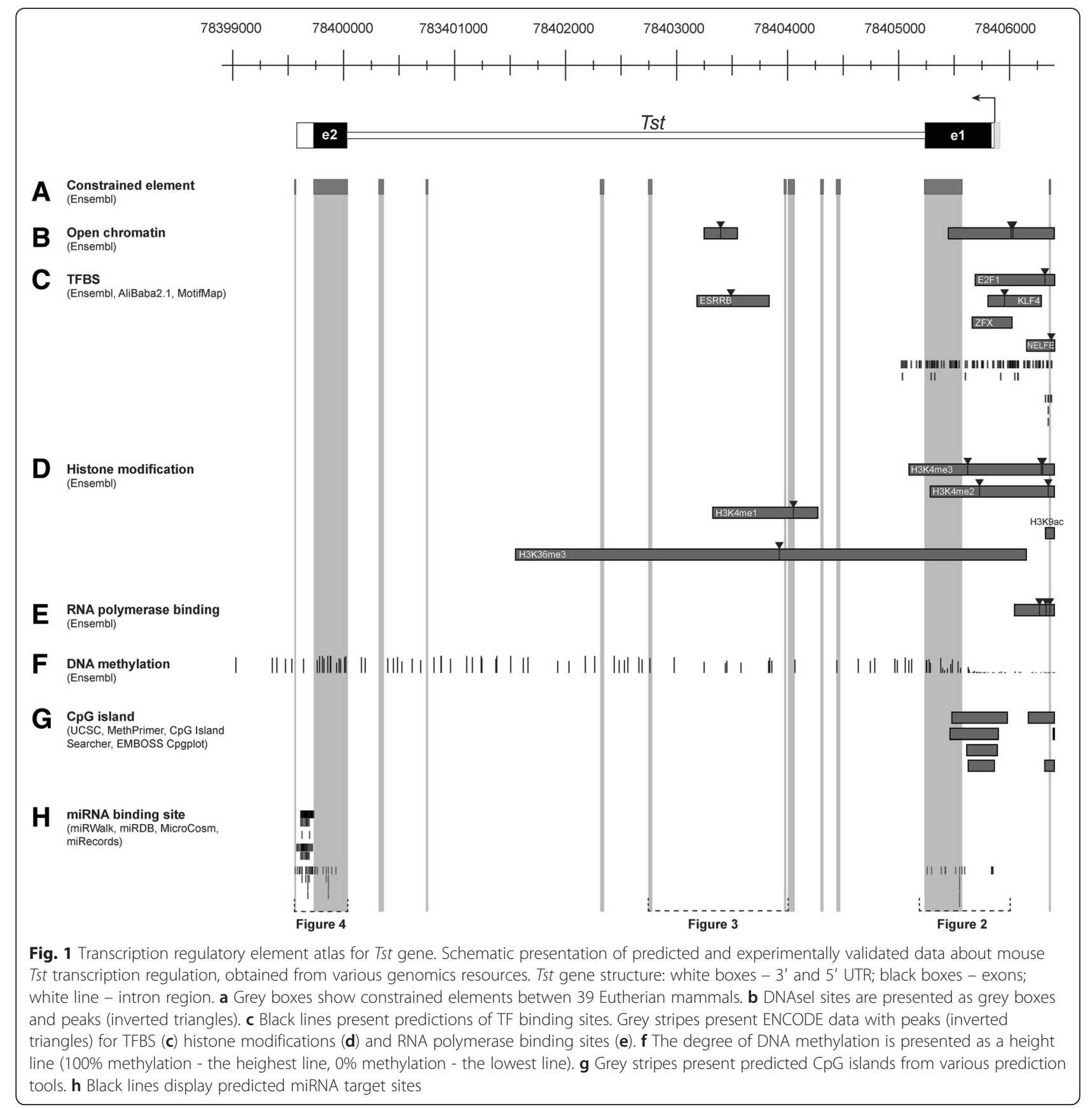

\section{Ensembl genome database and tools}

A 7400 bp-long segment of interest was analysed in the Ensembl database (Mouse genome coordinates:15:78399000 - 78406400) (release 79) [14]. Evolutionary constrained elements were extracted based on the nucleotide conservation between the 39 eutherian mammals in the "Location" tab under "Comparative Genomics - Alignments (text)" tree item. SNPs were categorized into variation consequence types - upstream gene variant, synonymous variant, intron variant, missense variant, nonsense variant, 5 ' or 3 ' untranslated region (UTR) variant and downstream gene variant. In the "Region in detail" tree item constrained elements for 39 eutherian mammals were obtained. DNA methylation state was indicated from dark blue (highly methylated), through green and then towards yellow (low methylation) and was based on the analysis in the mouse embryonic stem (ES) line. Data measuring chromatin activity or state (DNase I hypersensitive sites, histone modifications, polymerase II and III binding elements) and transcription factor binding sites were extracted and cell specific peaks were marked. Predicted miRNA targets from the Ensembl Regulation database were searched and displayed in the regulatory element map. 


\section{Eukaryotic promoter database (EPD)}

Experimentally validated eukaryotic RNA polymerase II promoter and TATA-box for Tst gene were retrieved from the EPD (EPDnew Mouse version 002) [23].

\section{AliBaba2.1}

An upstream sequence of the Tst gene (15:78405000 78406400) was analysed for predicted transcription factor binding sites using Alibaba2.1 program [24]. Default parameters settings were employed.

\section{MotifMap}

The MotifMap [25] system was used to obtain additional predictions of candidate regulatory elements. In the "Gene search" window, Tst gene was selected and searched for transcription factor binding sites in regions upstream and downstream of its transcription start site (TSS). All filters were kept as default, except upstream distance from TSS was set to $500 \mathrm{bp}$.

\section{CpG island prediction}

UCSC Genome Browser [26] and three additional web tools MethPrimer [27], CpG Island Searcher [28] and EMBOSS CpGplot [29] were used for searching CpG islands within the $0.5 \mathrm{~kb}$ DNA sequence upstream of the Tst gene. The islands are predicted by searching the sequence one base at a time, scoring each dinucleotide and identifying maximally scoring segments. These segments are then evaluated for the criteria: GC content $>50$, length $>200$ bp, observed/expected CpG ratio > 0.6 [30].

\section{MicroRNA miRNA target analysis}

miRNA target prediction tools (miRWalk [31], miRDB [32], MicroCosm [33], miRecords [34]) were used to identify potential binding sites for miRNAs within the 3' UTR and promoter of the Tst gene that could potentially function in Tst mRNA translatability.

\section{Sequencing the Tst locus in $\mathrm{F}$ and $\mathrm{L}$ lines}

Genomic DNA was isolated from spleen and liver of two mice per parental $\mathrm{F}$ and $\mathrm{L}$ line, using the procedures provided by the GenElute ${ }^{\mathrm{Tw}}$ Mammalian Genomic DNA Miniprep Kit (Sigma). DNA concentration was determined using a NanoVue Spectrophotometer (GE Healthcare Life Sciences). The $\sim 10 \mathrm{~kb}$ segment was divided in three overlapping smaller fragments (A, B, C; Additional file 11: Figure S2-A). PCR amplification was carried out using components from the Phusion High-Fidelity PCR Kit (Thermo SCIENTIFIC). The reaction mix contained 5x Phusion GC buffer, $10 \mathrm{mM}$ dNTPs, $10 \mu \mathrm{M}$ forward and reverse primers (Additional file 10: Table S10), $2 \mathrm{U} /$ $\mu \mathrm{l}$ Phusion DNA Polymerase and $50 \mathrm{ng} / \mu \mathrm{l}$ of gDNA in a total reaction volume of $20 \mu$ l. Thermocycler conditions were $98^{\circ} \mathrm{C}$ for $30 \mathrm{~s}$, followed by 35 cycles of $98^{\circ} \mathrm{C}$ for $7 \mathrm{~s}$ $68^{\circ} \mathrm{C}$ for $30 \mathrm{~s}$ and $72^{\circ} \mathrm{C}$ for $3 \mathrm{~min}$ and one cycle at $72^{\circ} \mathrm{C}$ for $10 \mathrm{~min}$. Samples were verified on $1.5 \%$ agarose gel and purified with GenElute ${ }^{\mathrm{rm}}$ PCR Clean-Up Kit (Sigma). Each amplified segment was then analysed in $10 \mu \mathrm{l}$ sequencing reactions, containing $20-80 \mathrm{ng} / \mu \mathrm{l}$ of PCR product and $5 \mu \mathrm{M}$ primers for detailed Sanger-sequencing (GATC Biotech). The MEGA 6 software [35] was used to assemble obtained sequences and to examine possible variations between the two mouse lines. Positions and direction of forward and reverse sequencing primers are displayed schematically in Figure S2-B (Additional file 11: Figure S2) whereas, nucleotide sequences of these primers are collated in Table S10 (Additional file 10: Table S10).

Ethics statement: DNA was isolated from mouse spleen and liver of two mice per parental $\mathrm{F}$ and $\mathrm{L}$ line at 14 weeks of age. Mice were humanely euthanised using $\mathrm{CO}_{2}$ followed by cervical dislocation. The use of animals to obtain mouse tissues for DNA isolation has been performed in accordance with the directive of the European Union 2010/63, approved by the Slovenian Ethical Committee for Animal Research and the Ministry of Agriculture, Forestry and Foods, Republic of Slovenia (licence No. U34401-55/2013/6).

\section{Results and discussion}

Evaluating how genomic variations in candidate genes for complex traits could affect the function and, consequently, the phenotype is a necessary step in positional cloning projects. Even when such projects result in identification of a gene responsible for the phenotypic effect we are still faced with a task of identifying the gene variant that is causal. This process, called quantitative trait nucleotide (QTN) identification, still presents a daunting task and can be specific for a particular gene or trait under study [36]. What is common to such projects is development of a priority list of gene variants for a particular candidate gene. The priority variant list is then used for validation in functional studies. Our general approach to build this integrative map is detailed in Additional file 12: Figure S1. In the first part of the study we here developed a bioinformatics approach to integrate existing knowledge into a regulatory element map of the Tst locus. In the second part, the entire Tst locus was sequenced between our mouse strains to uncover genetic variation. We then used the regulatory element map to prioritise likely causal Tst gene variants in the $\mathrm{F}$ and $\mathrm{L}$ lines.

\section{Locus-wide analysis of the Tst regulatory elements}

To construct an integrative map of important regulatory elements of the Tst locus, we employed various bioinformatics tools and databases as shown in the schematic overview in Additional file 12: Figure S1. Specifically, we searched for chromatin structure-related features such as histone modifications, open chromatin regions, potential 
DNA methylation sites, transcription factor, RNA polymerase and miRNA binding sites, as well as for genetic variants in all known sequenced mouse strains. As we used multiple available bioinformatics tools that often report or identify different elements or different size ranges for the same element, we decided to include all available information in a comprehensive map of Tst regulatory elements (Fig. 1).

The top panel of Fig. 1 shows the gene structure of the Tst gene. Ensembl database-derived reference sequence of the mouse Tst gene is $7400 \mathrm{bp}$-long and consists of 2 exons and one intron. The Ensembl or the UCSC Genome Browser do not provide annotations of any other overlapping protein or non-coding RNA gene in this segment, which was confirmed by our comprehensive bioinformatics analyses using other tools and prediction programs (see below). We next searched for evolutionary conservation of the $\sim 7.4 \mathrm{~kb}$ segment across a set of 39 eutherian mammals to identify constrained elements (Fig. 1a). Other regulatory features (Fig. 1b-h) overlapping with sequences of constrained elements are emphasized with a grey area in Fig. 1a. These constrained elements can serve as a guide to pinpoint regions of noncoding or coding DNA with conserved biological functions, and thus may be more likely to harbour SNP variants with functional consequences [37]. A total of 12 stretches of highest sequence conservation were identified amongst which the longest two regions covered the two exons, as expected. Nine of 12 highly conserved regions were identified also in the nonexonic sequences, eight in the intron and one in the flanking sequence upstream of the Tst promotor. However, our result of eight conserved blocks presenting $5 \%$ of intronic sequence suggests that the Tst intron contains fewer highly conserved elements compared with $12-28 \%$ found in three mammalian orders [38].

The formation of open chromatin in eukaryotic genomes is an important factor controlling potential regulatory activity [39]. Such active functional elements can be located through the identification of regions of the genome that are hypersensitive to DNase I cleavage [40], so called DNase I hypersensitive sites or regions of open chromatin. The ENCODE project uncovered two open chromatin sites for the mouse Tst gene (Fig. 1b). The proximal DNase I hypersensitive site was found within the intron. A larger DNAse I hypersensitive site was located distally and overlapped the Tst promoter. The existence of functional open chromatin sites is corroborated by mapping TF binding sites within these regions using two different transcription factor binding sites (TFBS) analyses algorithms (Alibaba2.1 and Motifmap) and Ensembl database annotations (Fig. 1c; Additional file 7: Table S7). Additionally, Ensembl database annotated various histone modification regions (Fig. 1d) to the two aforementioned open chromatin regions. RNA polymerase II binding sites have been experimentally demonstrated in two different cell lines examined by ENCODE within the promoter open chromatin region (Fig. 1e). Therefore, several lines of evidence support the existence of two Tst open chromatin regions with a regulatory role, a smaller intronic region and a larger promoter open chromatin region.

DNA methylation marks potential sites for epigenetic regulation found to be important in many biological processes especially in gene expression regulation. We found $118 \mathrm{CpG}$ sites in the examined 7.4 kb Tst genome segment. Cytosine methylation varied from $0 \%$ (unmethylated) to $100 \%$ (methylated), which is displayed (Fig. 1f) as height bars. Methylation of large CGrich stretches of DNA especially in promoters strongly correlates with suppression of gene expression [41]. We used various tools to identify $\mathrm{CpG}$ islands (Fig. 1g) which all predicted two clusters of non-methylated CpG islands. This segment co-localized with the predicted promoter, indicating its potential role in transcriptional regulation of Tst. Micro RNA miRNA binding site prediction tools (Fig. 1h) provide strong evidence for the existence of miRNA regulatory sites within the 3'UTR located in the second Tst exon (Fig. 1h).

Therefore, a comparative analysis of various regulatory features provides strong evidence for existence of three regulatory genome segments. These locate to the promoter, intron and 3'UTR of the Tst locus (marked at the bottom of Fig. 1) and will be explored in more detail in the following sections and Figs. 2, 3 and 4).

\section{Regulatory features of the Tst core promoter and exon 1 region}

To define the location of the core promoter sequences we used the Eukaryotic promoter database (EPD) tools that located it within a 59 bp-long interval overlapping the transcription initiation site (TSS, Fig. 2a - black). No TATA-box or CG box consensus sequences were identified around this core promoter segment. Genetic variation in the mouse genome in this segment identified 14 SNPs, one of which mapped to the evolutionary constrained element (Fig. 2b) and one SNP to the core promoter (Fig. 2c, presented as red lines). SNPs occurring in highly conserved functional regions may affect expression and phenotypic variability in strains harbouring the mutant alleles. The open chromatin region (Fig. 2d) harbours four transcription factor binding sites (TFBS) for transcription factors ZFX, E2F1, KLF4 and NELFE (Fig. 2e). Ensembl annotates these TFBS from ChIP-Seq experiments in selected cell lines (ES and MEL) so it is possible that some TFs are missed as they don't regulate TST in the examined cell lines and/ or act in vivo in a tissue specific manner. For this reason we applied two additional tools, Alibaba2.1 and MotifMap, that predicted a variety of different transcription factors 


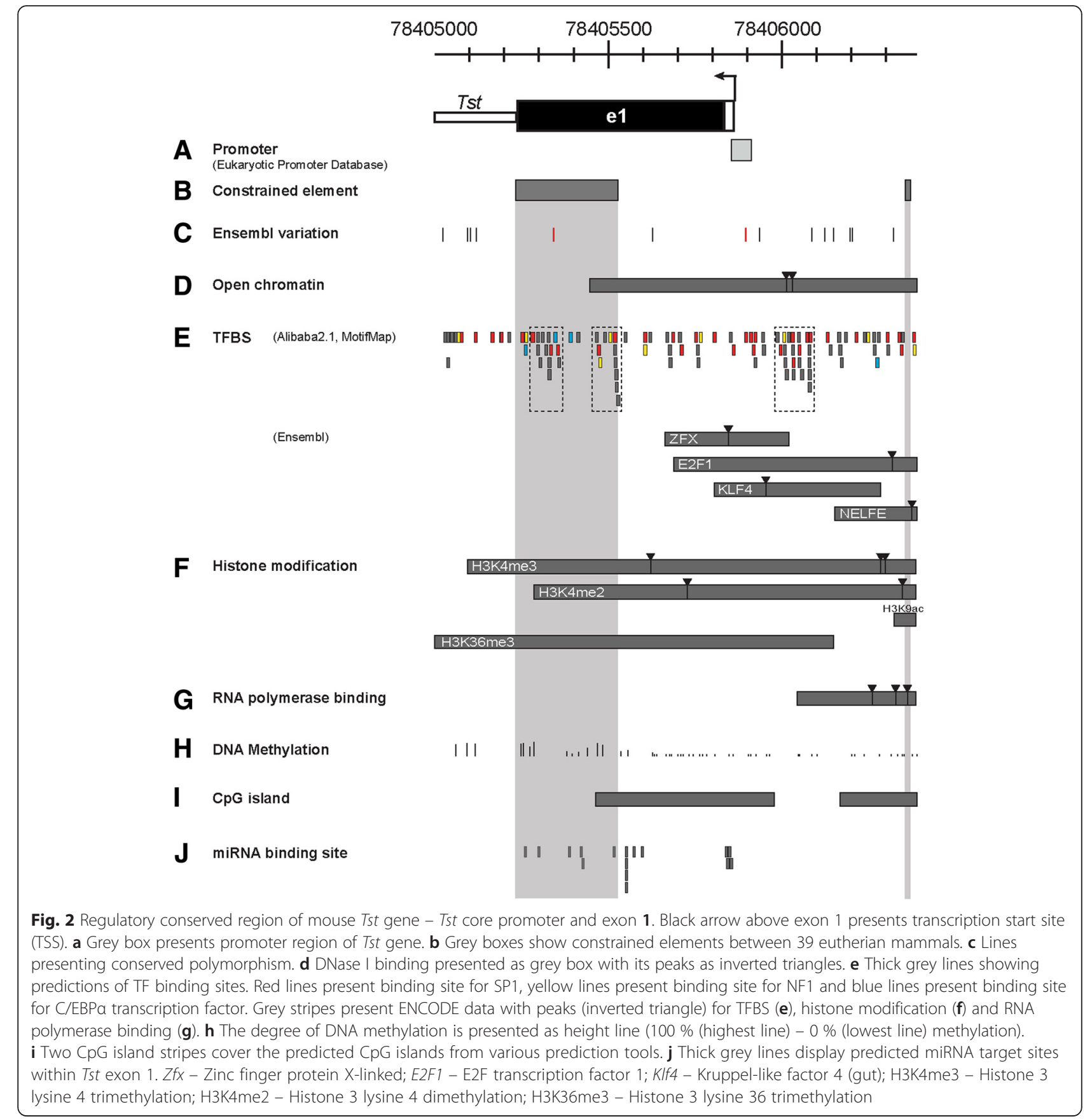

(Fig. 2) potential binding sites $(n=55)$ in the region of open chromatin (Fig. 2d-e; Additional file 7: Table S7) as well as constrained element (Fig. 2b). One has to be cautious about all these predicted TFBS as they are not experimentally verified and do not tell us anything about tissue specificity. However, the SP1 binding site was frequently predicted (Fig. 2e, red thick line) and this TF has already been linked as a cellular glucose sensor and it is also involved in leptin promoter activity [42]. In line with this is our observation that SP1 sites overlap with open chromatin region. In addition, Alibaba and
MotifMap algorithms frequently predicted sites for NF1 (Fig. 2e, yellow thick line), a transcription activator binding protein [43] and $\mathrm{C} / \mathrm{EBP} \alpha$ (Fig. 2e, blue thick line), a liver-enriched transcriptional regulator involved in energy metabolism [44]. In the context of Tst regulation, none of these sites have actually been experimentally validated. However, TFBS are clustered in three small segments (dashed box in Fig. 2e), two within the proximal constrained element and the third on top of the peak of open chromatin site (between 78406000 - 78406100). Additionally, TFBS overlap with the less methylated 


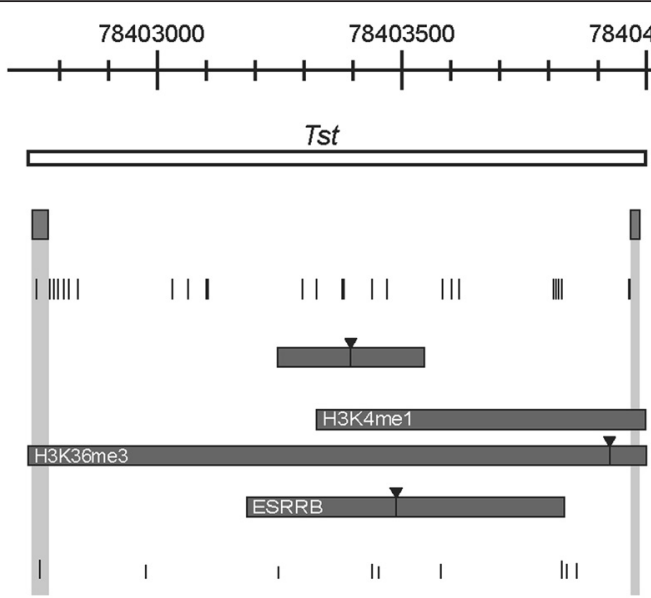

Fig. 3 Regulatory conserved region of mouse Tst gene - intron. a grey boxes show constrained elemnts between 39 Eutherian mammals. b Lines present conserved polymorphisms. Stripes present data froom ENCODE project with peaks (inverted triangles) for (c) open chromatin, (d) histone modifications and (e) TFBS. f The degree of DNA methylation is presented as a height line (100\% methylation is the highest line, while $0 \%$ methylation is the lowest line). H3K4me1 - Histone 3 lysine 4 methylation; H3K36me3 - Histone 3 lysine 36 trimethylation; Esrrb - Estrogen related receptor beta

CpG sites (Figs. H, I), open chromatin, RNA polymerase binding sites and histone modification motifs specific for active transcription. Therefore, our analysis of TFBS offers grounds and directions for further experimental work to examine which TFBS are functional for tissue-specific Tst regulation.

Three different histone modifications (Fig. 2h) were identified in this segment: H3K4me3 - one of the most studied chromatin modifications, present at actively transcribed protein coding promoters in eukaryotes; H3K4me2 - these modifications are enriched within TFBS sites [45]; and H3K36me3 - enrichment of these modifications was found to be higher at exonic than intronic regulatory sites within the Tst locus, which is in line with previous reports [46]. We conclude that the Tst core promoter, $5^{\prime}$ UTR and exon 1 region contain most types of histone modifications typically found in promoters of actively expressed protein coding genes.

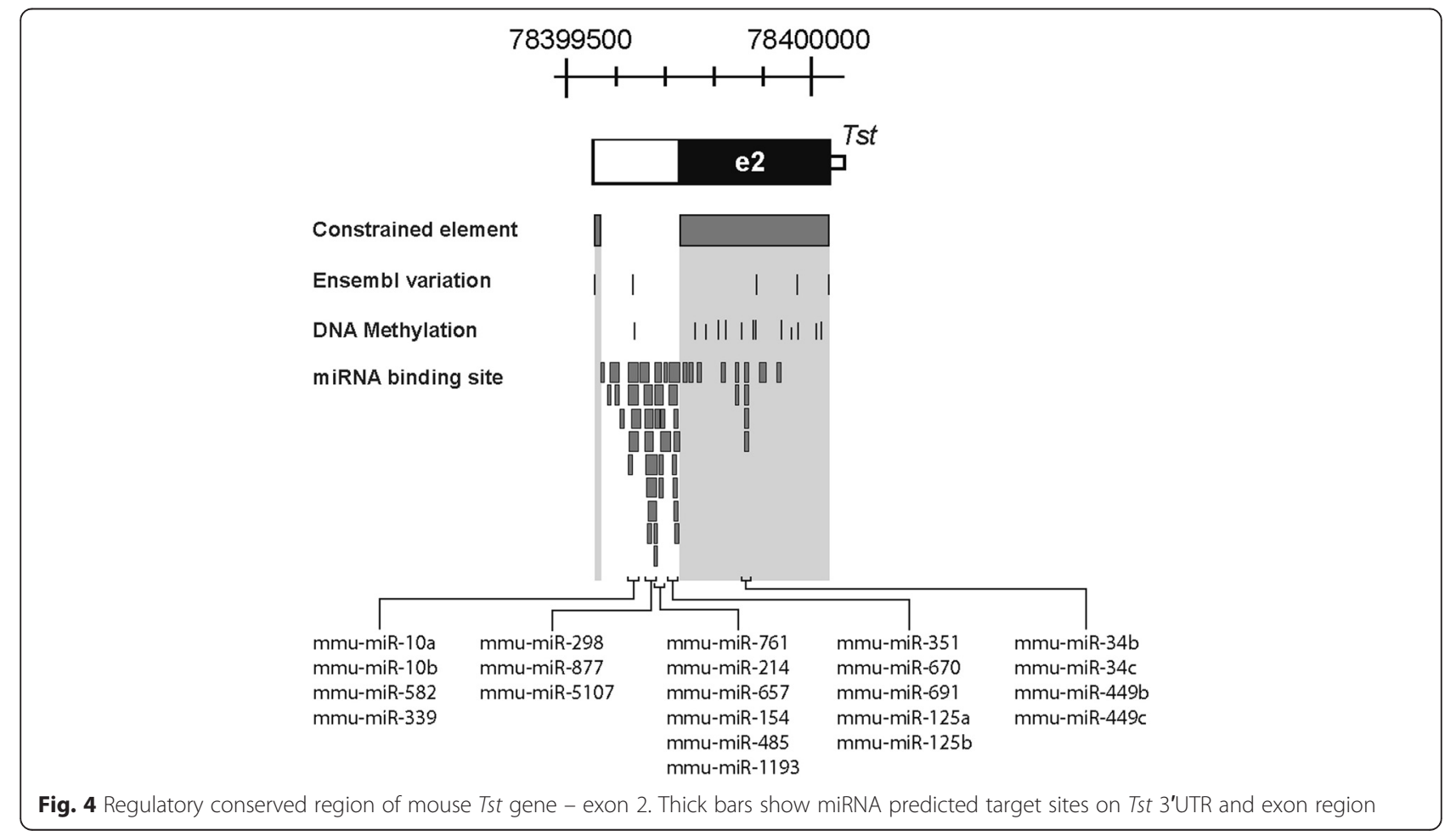




\section{Regulatory features of the Tst intronic region}

Our bioinformatics analysis revealed strong evidence for the existence of the second regulatory region within intron of the Tst gene (Fig. 1). Introns can affect transcription by acting as repositories for transcriptional regulatory elements (i.e. enhancers and repressors). We narrowed a segment potentially important in the regulation of Tst to a region between $78402750-7840000 \mathrm{bp}$. Using different tools and databases we identified regulatory elements or features such as constrained elements (Fig. 3a), open chromatin site (Fig. 3c), two histone modifications specific for transcribed portions of genes and associated with enhancers and other distal elements (Fig. 3d), and also one TFBS (Fig. 3e). Additionally, the frequency of DNA methylation sites (Fig. 3f) was statistically significantly reduced in this segment when compared with the frequency of DNA methylation in the rest of the intron (Chi-square test, p-value $<0.01$ ). Overlapping regulatory intronic features such as open chromatin, histone modification H3K4me1 and the binding site for transcription factor estrogen related receptor, beta (ESRRB) could indicate the presence of a functional intragenic enhancer for Tst or flanking genes, as was shown for some other genes [47]. Direct involvement of ESRRB in the regulation of Tst gene has not yet been demonstrated. However, binding of ESRRB to a consensus TFBS as the one found in intron of Tst, was experimentally mapped in the mouse ES cells which also supported previous genetic studies [48-50] suggesting its role in the self-renewal of ES cells. Of note, for the context of our study and the Fat and Lean model of mice, is the study of $\mathrm{Li}$ et al. [51] that achieved full reprogramming of nave-like porcine adipose induced stem cells and linked this process, in part, by significant up-regulation of ESRRB. Transcriptomics studies also determined RNA expression of Esrrb in adipocytes [52]. Therefore, our results suggest that ESRRB could be regulating abundance of TST protein in a tissue (adipose) specific manner by using the identified binding -enhancer site in intron of Tst.

\section{Regulatory features of the Tst $3^{\prime} U T R$ region}

We positioned the third important regulatory region in the second exon of the Tst gene (Fig. 4). Approximately half of the distal part of this exon contains the 3'UTR region that is frequently found to regulate gene expression at the post-transcriptional level in eukaryotes [53]. Regions conserved and important for such regulation are around a poly(A) tail consensus site ( $5^{\prime}$-AATAAA- $3^{\prime}$ ), found between 78399577 - 78399572 bp which in cooperation with poly(A) binding proteins, contributes to regulation of mRNA translation, stability and export. It could also be possible that the structural characteristics of the 3'UTR may contribute to gene expression, as in general, longer 3'UTR correlate with lower expression rates, since they contain more miRNA and protein binding sites that are involved in inhibiting translation. We searched for miRNA binding sites using various prediction tools such as miRWalk [31], miRDB [32], MicroCosm [33], and miRecords [34]. As shown in Fig. 4 there is a considerable overlap of hits by these various tools providing strong support for existence of miRNA sites in the 3'UTR of Tst. Two clusters emerged, one in the coding sequence of exon 2 and a more prominent miRNA binding region in the 3'UTR. A total of 54 different miRNA were predicted to bind four sites within 3'UTR, among which mmu-miR-10a, mmu-miR-10b, mmu-miR-761, mmu-miR-214, mmumiR-670, mmu-miR-877 and mmu-miR-339 were predicted by almost all used tools. That mammalian 3' UTR regions can be targeted by multiple miRNAs has recently been demonstrated in a large-scale genomic screen of miRNA-mRNA interactions [54]. That the predicted four miRNA binding sites in the 3'UTR of Tst may indeed be functional is supported by reduced genetic variation in these sites among 15 mouse strains. Only one miRNA binding site was found to be variable with a non-reference nucleotide found only in 4 out of 15 strains as will be explained in more detail in the next section. Furthermore, the location and structural features of the four sites indicate that the sites may indeed be functional and efficient. Sites are positioned at least 15 nucleotides downstream of the stop codon and are close to one end of 3'UTR, which are both strong features of effective miRNA binding sites as found in an experimental study of Grimson et al. [55]. The four sites also exhibit AU-richness and lie in unstructured areas, which were experimentally found to correlate with increased accessibility for the miRNA regulatory complex [56]. Therefore, on the basis of our bioinformatics analysis that identified several potential miRNA binding sites whose target site location and sequence context imply functional efficacy. We can conclude that the Tst locus is likely to be rich in miRNA target sites and hence likely to be regulated by miRNAs to affect expression or tissue specificity.

\section{Identifying and prioritising polymorphisms of the Fat and Lean lines using the regulatory element $T s t$ map Sequence analysis of Tst locus in $F$ and $L$ lines}

Once the integrative map of potentially functional regulatory elements within the Tst locus was constructed (Figs. 1, 2, 3 and 4 above), we used it for prioritising genetic variants potentially causal for the Tst in our obesity/ leanness mouse lines. To accomplish this, we first needed a high resolution sequence of the Tst locus in both lines to identify all possible polymorphisms. The sequencing strategy and locus view of 10962 bp-long 
sequenced segment (Chr 15: 78399030 - 78409992 bp) covering the Tst gene plus additional 4133 bp sequence of the promoter (Chr15: 78405859 - 78409992) and 526 bp downstream of the $3^{\prime}$ end of the gene (Chr15: 78399556 - 78399030) is shown in Additional file 11: Figure S2. The aligned sequences between the F and L mouse lines revealed only five polymorphisms (Fig. 5a). Considering the length of the mouse genome and a total number of short variants one would expect on average 39.4 bp per variant. With only five variants between the Fat and Lean line this rate is significantly reduced to about one variant per $1050 \mathrm{bp}$ indicating that this genomic region in $\mathrm{F}$ and $\mathrm{L}$ mice is likely derived from two closely related progenitor lines. In comparison with the reference sequence of the mouse genome (strain C57BL/6J) the Fat and Lean lines contained 4 SNPs, a one nucleotide deletion and a dinucleotide insertion in the Fat line (Fig. 5b). Apart from SNP rs31534689 that resides in the 3'UTR of the second Tst exon and rs251994838 located in a constrained element, all other gene variants mapped to the intron whereas none were identified in $\sim 5 \mathrm{~kb}$ segment upstream of transcription start site. The sequence analysis of the Fat and Lean lines revealed that no private alleles, i.e. specific to Fat or Lean line only, exist, which is in line with previous data demonstrating that only $\sim 2 \%$ private variants exist across the whole genomes of laboratory strains of mice [57]. Sequencing of the 10962 bp-long segment covering the Tst locus in the F and L lines identified five polymorphisms providing a basis for further comparative genomic analyses and prioritisation of potential functional variants.

\section{Strain of origin of the Tst region in the $F$ and $L$ lines}

Once we had a complete and high resolution sequence of the Tst locus from the Fat and Lean lines we asked if we can identify from which mouse strain, species or subspecies is this locus derived from in Fat and Lean mice. As alluded in the introduction, Fat and Lean lines were selected for more than 60 generations for high or low body fat $\%$ from a common base population derived from crosses of two inbred lines JU and CBA and an outbred strain CFLP from the Carnworth laboratory [15]. As none of the samples from these original base population strains are available, it is not possible to definitively ascertain the origin of the Tst locus DNA in our current Fat and Lean inbred lines. However, on the basis of our new sequence data we can predict the likely origin from comparative sequence and haplotype analysis. The Fat line shared the exact haplotype of all gene variants only with the $\mathrm{WSB} / \mathrm{EiJ}$ strain but was also very similar (four of five variants in common) with other wild-derived mouse strains such as CAST/EiJ, PWK/PhJ

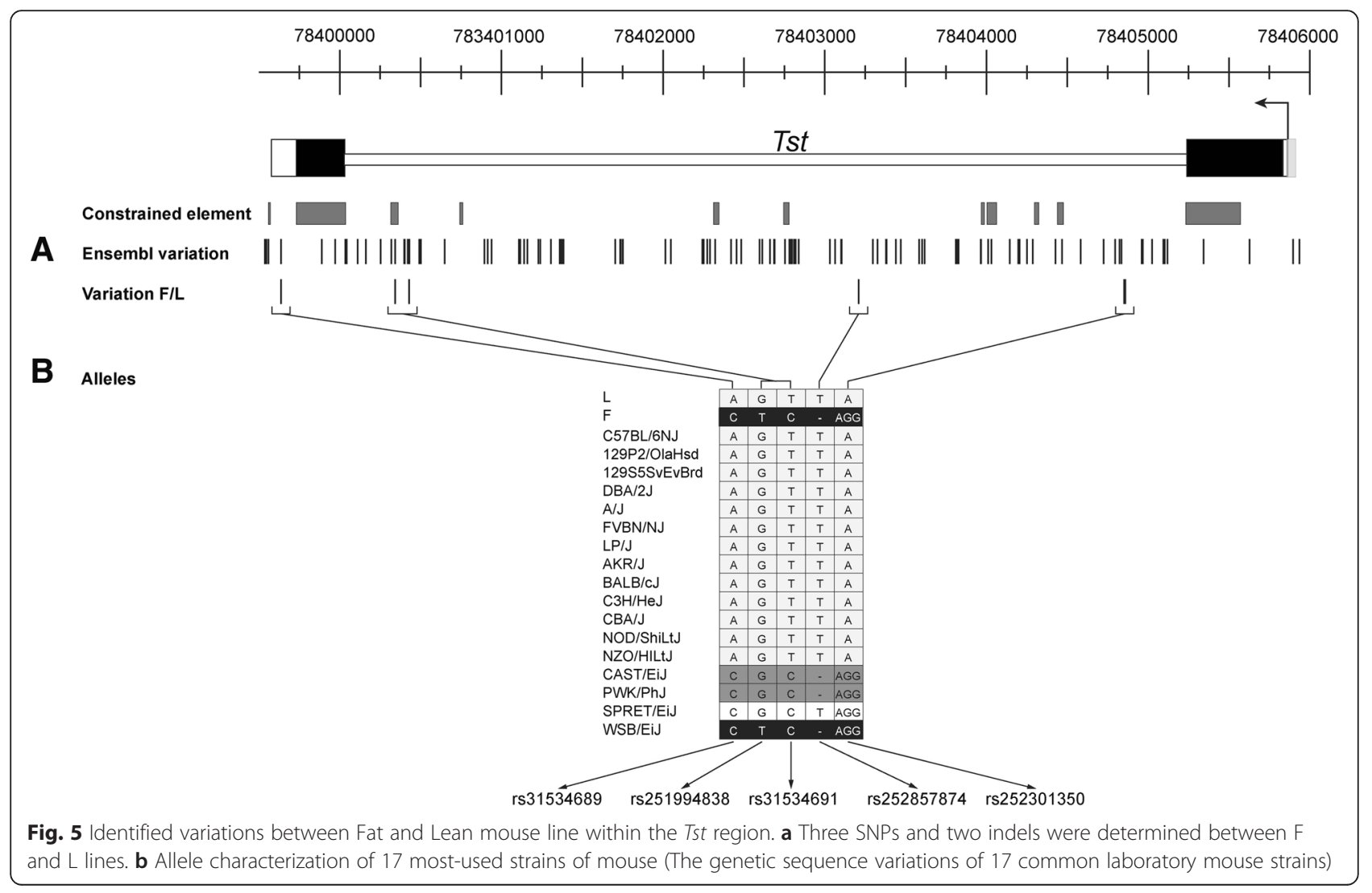


and SPRET/EiJ. Since the wild derived WSB/EiJ strain is a representative of Mus musculus domesticus subspecies [57] we conclude that the Fat line also contains the $M$. $m$. domesticus DNA at the Tst locus. The Lean line shared a haplotype for the five variants with 13 other classical inbred mouse strains including the reference C57BL/6J strain (Fig. 5b). As demonstrated before [58], these classical laboratory strain genomes are mosaics of genomes from four taxa, Mus musculus castaneus, Mus musculus musculus, Mus musculus domesticus and Mus spretus. The mouse genome, in contrast to human, where projects like 1000-human genomes have already been accomplished, lacks comprehensive data on variation of natural mouse populations from which the classical laboratory strains were derived [59]. It is thus not possible to assign local ancestry at a finer scale and this is the case for the Tst locus in the Lean line. However, our sequencing and haplotype analyses results do suggest that the Fat line Tst locus has a "wild type" haplotype and the Lean line allele is of the inbred laboratory strain origin. This is in line with our previous transcriptomics results demonstrating overexpression of Tst in the Lean line over the comparator ("wild type" Tst) expression of the Fat line [22]. It is likely that one, or a combination of the five identified genetic variants are responsible for this overexpression effect.

\section{Prioritisation of genetic variants in relation to the Tst regulatory element map}

As our final goal is to identify the causal genetic variant for the phenotypic effect on leanness/obesity of the Tst locus it is important to prioritise potential candidate genetic variants before embarking upon more focused and lengthy functional analyses downstream. As the majority of genetic variants in genome-wide association studies in humans and animal models as well as re-sequencing projects are located in non-coding regions, this hinders the obvious assessment of their functional effect as the protein sequence itself is not modified.

Here we combined our bioinformatics-based regulatory atlas of the Tst locus and identified genetic variants between our target strains to prioritise them functionally and hence improve our chances to select potential causal variants for further experimental work.

Non-coding SNPs can have large cis-regulatory effects if they lie in important regulatory DNA motifs via, for example, altering their affinity for TFs, splicing procedures or chromatin remodelling processes. In our case, only one intronic variant, rs251994838, overlapped with an evolutionary constrained intronic element and could hence have a regulatory impact on the Tst gene expression by affecting DNA accessibility (chromatin structure) or the affinity of transcription factors binding. The second high priority genetic variant candidate (rs31534689) lies in the 3'UTR (Fig. 6). Our bioinformatics analysis identified a large cluster of potential miRNA binding sites around this genetic variant using various miRNA binding site prediction tools. Amongst potential miRNA species, miRNA mmu-miR338-5p is predicted to bind to the 3'UTR Lean line allele with no mismatches and with a mismatch to the

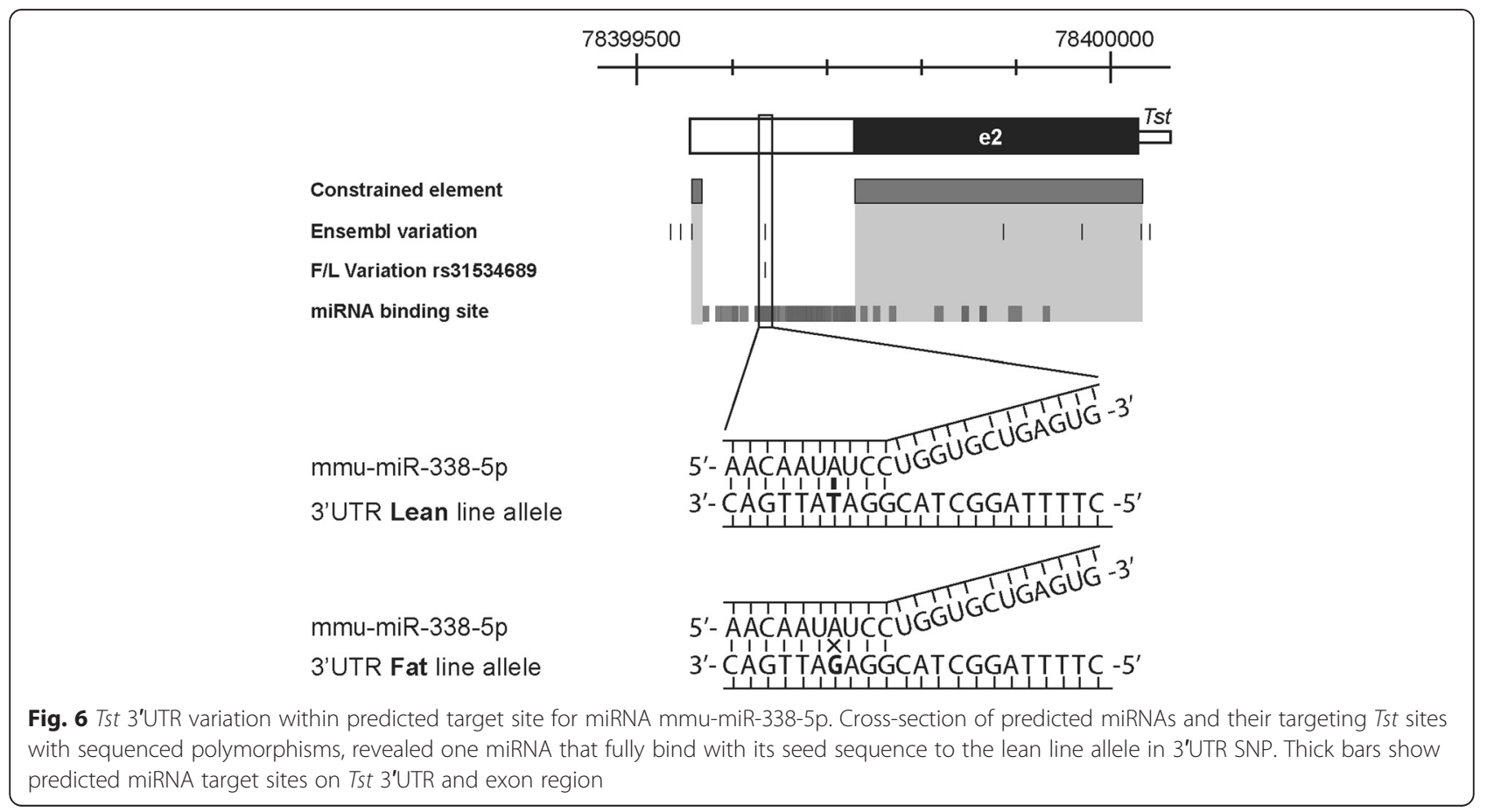


Fat line allele (Fig. 6). The rs31534689 polymorphism is located in a so-called seed region of miRNA [60], where complete complementarity plays a major role in miRNA target recognition. This SNP therefore has a potential to affect differential Tst mRNA stability or translatability. We conclude that rs251994838 and rs31534689 represent the highest priority candidate genetic variants for further experimental functional validation of their causality on the phenotype. As Tst was found to be overexpressed in the Lean line specifically in the white adipose tissue [61], such validations will likely have to be carried out in the context of adipocyte cell lines or white adipose tissue. New CRISPR-based transgenesis approaches also offer promising tools to evaluate causality by allele replacement approaches in vivo.

\section{Conclusions}

In our recent positional cloning experiment we identified Tst as the causal gene for the Fob3b2 QTL phenotypic effect on leanness with improved metabolic parameters [22]. As increased Tst expression selectively in adipose tissue was found responsible for this effect, we focused here on developing a map of regulatory elements for the Tst locus in mice potentially involved in regulating the mRNA transcript levels encoding this enzyme. The map provides a basis for planning further experimental validations and functional analyses of this important and evolutionary conserved gene. It helped to narrow down Tst genetic variants to a small testable list of candidate polymorphisms for more focused and hypothesis-driven experimental work. Our approach combined information on evolutionary constrained elements, promoter, enhancer, epigenetic and chromatinrelated regulatory features, protein and ncRNA binding sites and genetic variants into a single integrated regulatory element map. This approach, demonstrated here for the Tst locus, is of general utility and could be used to develop regulatory element maps for other genes also in other species to classify, prioritize and functionally interpret potentially causal regulatory genetic variation. As most complex trait and disease associations detected by genetic studies lie outside coding regions, developing a regulatory element map in candidate genes could inform which annotations of gene structures and regulatory elements contain likely causal variants in the target gene under study. This can lead to identification of regulatory variants and interpretation of how variation in regulatory mechanisms results in controlling gene expression or activity. A map of regulatory elements described in this study can therefore provide additional layer of biologically-relevant information to the genome sequence itself and serve as the basis for post-GWAS or post-QTL functional studies.

\section{Additional files}

Additional file 1: Table S1. Constrained elements for 39 eutherian mammals (Ensembl). (DOCX $15 \mathrm{~kb}$ )

Additional file 2: Table S2. Sites enriched for marks of open chromatin (Ensembl). (DOCX $15 \mathrm{~kb}$ )

Additional file 3: Table S3. RNA polymerase binding sites (Ensembl). (DOCX $15 \mathrm{~kb}$ )

Additional file 4: Table S4. CpG islands - genome coordinates. (DOCX $15 \mathrm{~kb})$

Additional file 5: Table S5. Histone modifications (Ensembl). (DOCX 16 $\mathrm{kb})$

Additional file 6: Table S6. DNA methylation in Embryonic stem cell. (DOCX 19 kb)

Additional file 7: Table S7. Sites enriched for marks of transcription factor binding sites. (DOCX $23 \mathrm{~kb}$ )

Additional file 8: Table S8. Predicted miRNA target sites. (DOCX 22 kb)

Additional file 9: Table 59. Merged mouse single nucleotide polymorphisms (SNP) from Ensembl and Mouse genomes project (Wellcome Trust Sanger Institute). (DOCX 63 kb)

Additional file 10: Table S10. Sequences of primers used in sequencing of Tst locus (region). (DOCX $17 \mathrm{~kb}$ )

Additional file 11: Figure S2. Primers positions used in sequencing the Tst locus. A) Positions of primers used for amplification of $\sim 3 \mathrm{~kb}$ segments $(A, B, C)$ of Tst region. B) Location of $P C R$ primers for detailed sequencing of $A, B$, and $C$ segments. (JPG $4083 \mathrm{~kb}$ )

Additional file 12: Figure S1. Integrated gene transcription regulatory elements for atlas development. Schematic of elements affecting gene transcription and expression at the level of chromatin state (histone modifications, DNA methylation, chromatin accessibility), through transcription factors and RNA polymerase binding, variation impact and microRNAs miRNAs influence. (JPG $1035 \mathrm{~kb}$ )

\section{Abbreviations}

F, fat line; gDNA, genomic DNA; GWAS, genome-wide association study; L, lean line; miRNA, micro RNA; $\mathrm{PPCR}$, quantitative polymerase chain reaction; QTL, quantitative trait loci; RT, reverse transcription; SNPS, single nucleotides polymorphisms; Tst, thiosulfate sulfurtransferase

\section{Acknowledgements}

We thank Vesna Mrak for technical assistance in the laboratory.

\section{Funding}

This study was funded by grants from the Slovenian Research Agency: core funding program P4-0220, J3-6804, and Young Investigator Grant for Jasmina Beltram. N.M. Morton was supported by an Investigator Award from the Wellcome Trust (100981/Z/13/Z) The funders had no role in the design of the study and collection, analysis, decision to publish, interpretation of data or preparation of the manuscript.

\section{Availability of data and materials}

The datasets supporting the conclusions of this article are included in Fig. 5 and Additional file 9: Table S9 and Additional file 10: Table S10.

\section{Authors' contributions}

$J B$ participated in the design of the study, carried out the sequencing procedures, performed the data analysis and wrote the manuscript. SH participated in designing the study and wrote the manuscript. NMM and TK were involved in data interpretation and drafting the manuscript. All authors have read and approved the final version of the manuscript.

\section{Competing interests}

The authors declare that they have no competing interests.

Consent to publish

Not applicable. 


\section{Ethics approval and consent to participate}

The use of animals to obtain mouse mouse tissues for DNA isolation has been performed in accordance with the directive of the European Union 2010/63, approved by the Slovenian Ethical Committee for Animal Research and the Ministry of Agriculture, Forestry and Foods, Republic of Slovenia (licence No. U34401-55/2013/6).

\section{Author details}

'Biotechnical Faculty, Animal Science Department, University of Ljubljana, Groblje 3, 1230 Domzale, Slovenia. 'Molecular Metabolism Group, University/ British Heart Foundation Centre for Cardiovascular Science, University of Edinburgh, Queen's Medical Research Institute, 47 Little France Crescent, Edinburgh EH16 4TJ, UK. ${ }^{3}$ National Institute of Chemistry, Hajdrihova 19, 1000 Ljubljana, Slovenia.

Received: 20 February 2016 Accepted: 25 May 2016

Published online: 11 June 2016

\section{References}

1. Welter D, MacArthur J, Morales J, Burdett T, Hall P, Junkins H, Klemm A, Flicek P, Manolio T, Hindorff L, Parkinson H. The NHGRI GWAS Catalog, a curated resource of SNP-trait associations. Nucleic Acids Res. 2014; 42(Database issue):D1001-6.

2. 1000 Genomes Project Consortium, Abecasis GR, Auton A, Brooks LD, DePristo MA, Durbin RM, Handsaker RE, Kang HM, Marth GT, McVean GA. An integrated map of genetic variation from 1,092 human genomes. Nature. 2012:491:56-65.

3. International HapMap 3 Consortium, Altshuler DM, Gibbs RA, Peltonen L Altshuler DM, Gibbs RA, et al. Integrating common and rare genetic variation in diverse human populations. Nature. 2010;467:52-8.

4. Raychaudhuri S. Mapping rare and common causal alleles for complex human diseases. Cell. 2011;147:57-69.

5. Eppig JT, Blake JA, Bult CJ, Kadin JA, Richardson JE, Mouse Genome Database Group. The Mouse Genome Database (MGD): facilitating mouse as a model for human biology and disease. Nucleic Acids Res. 2015; 43(Database ossue):D726-36

6. Swanberg $M$, Lidman $O$, Padyukov L, Eriksson $P$, Akesson $E$, Jagodic $M$ Lobell A, Khademi M, Börjesson O, Lindgren CM, Lundman P, Brookes AJ Kere J, Luthman H, Alfredsson L, Hillert J, Klareskog L, Hamsten A, Piehl F, Olsson T. MHC2TA is associated with differential MHC molecule expression and susceptibility to rheumatoid arthritis, multiple sclerosis and myocardial infarction. Nat Genet. 2005;37(5):486-94.

7. Aitman TJ, Dong R, Vyse TJ, Norsworthy PJ, Johnson MD, Smith J, Mangion J, Roberton-Lowe C, Marshall AJ, Petretto E, Hodges MD, Bhangal G, Patel SG, Sheehan-Rooney K, Duda M, Cook PR, Evans DJ, Domin J, Flint J, Boyle $\mathrm{J}$, Pusey CD, Cook HT. Copy number polymorphism in Fcgr3 predisposes to glomerulonephritis in rats and humans. Nature. 2006;439(7078):851-5.

8. ENCODE Project Consortium. An integrated encyclopedia of DNA elements in the human genome. Nature. 2012;489(7414):57-74.

9. Bono H, Kasukawa T, Furuno M, Hayashizaki Y, Okazaki YFANTOMDB. Database of functional annotation of RIKEN mouse cDNA clones. Nucleic Acids Res. 2002;30(1):116-8.

10. Bernstein BE, Stamatoyannopoulos JA, Costello JF, Ren B, Milosavljevic A, Meissner A, Kellis M, Marra MA, Beaudet AL, Ecker JR, Farnham PJ, Hirst M, Lander ES, Mikkelsen TS, Thomson JA. The NIH Roadmap Epigenomics Mapping Consortium. Nat Biotechnol. 2010;28(10):1045-8.

11. Ravasi T, Suzuki H, Cannistraci CV, Katayama S, Bajic VB, Tan K, Akalin A, Schmeier S, Kanamori-Katayama M, Bertin N, Carninci P, Daub CO, Forrest AR, Gough J, Grimmond S, Han JH, Hashimoto T, Hide W, Hofmann O, Kamburov A, Kaur M, Kawaji H, Kubosaki A, Lassmann T, van Nimwegen E, MacPherson CR, Ogawa C, Radovanovic A, Schwartz A, Teasdale RD, Tegnér J, Lenhard B, Teichmann SA, Arakawa T, Ninomiya N, Murakami K, Tagami M, Fukuda S, Imamura K, Kai C, Ishihara R, Kitazume Y, Kawai J, Hume DA, Ideker T, Hayashizaki Y. An atlas of combinatorial transcriptional regulation in mouse and man. Cell. 2010;140(5):744-52.

12. Yan J, Enge $M$, Whitington $T$, Dave $K$, Liu J, Sur I, Schmierer B, Jolma A, Kivioja T, Taipale M, Taipale J. Transcription factor binding in human cells occurs in dense clusters formed around cohesin anchor sites. Cell. 2013; 154(4):801-13.
13. Guttman M, Amit I, Garber M, French C, Lin MF, Feldser D, Huarte M, Zuk O, Carey BW, Cassady JP, Cabili MN, Jaenisch R, Mikkelsen TS, Jacks T, Hacohen N, Bernstein BE, Kellis M, Regev A, Rinn JL, Lander ES. Chromatin signature reveals over a thousand highly conserved large non-coding RNAs in mammals. Nature. 2009;458(7235):223-7.

14. Flicek $P$, Amode MR, Barrell D, Beal K, Billis K, Brent $S$, Carvalho-Silva D, Clapham P, Coates G, Fitzgerald S, Gil L, Girón CG, Gordon L, Hourlier T, Hunt S, Johnson N, Juettemann T, Kähäri AK, Keenan S, Kulesha $E_{1}$ Martin FJ, Maurel T, McLaren WM, Murphy DN, Nag R, Overduin B, Pignatelli M, Pritchard B, Pritchard E, Riat HS, Ruffier M, Sheppard D, Taylor K, Thormann A, Trevanion SJ, Vullo A, Wilder SP, Wilson M, Zadissa A, Aken BL, Birney E, Cunningham F, Harrow J, Herrero J, Hubbard TJ, Kinsella R, Muffato M, Parker A, Spudich G, Yates A, Zerbino DR, Searle SM. Ensembl. Nucleic Acids Res. 2014;42(Database issue):D749-55. http://www.ensembl.org/index.html. Accessed 27 Apr 2015.

15. Sharp GL, Hill WG, Robertson A. Effects of selection on growth, body composition and food intake in mice. I. Responses in selected traits. Genet Res. 1984;43(1):75-92.

16. Bünger $L$, Hill WG. Inbred lines of mice derived from long-term divergent selection on fat content and body weight. Mamm Genome. 1999;10(6):645-8.

17. Horvat S, Bünger L, Falconer VM, Mackay P, Law A, Bulfield G, Keightley PD. Mapping of obesity QTLs in a cross between mouse lines divergently selected on fat content. Mamm Genome. 2000;11(1):2-7.

18. Brockmann GA, Bevova M. Using mouse models to dissect the genetics of obesity. Trends Genet. 2002;18(7):367-76.

19. Spiegelman BM, Flier JS. Obesity and the regulation of energy balance. Cell. 2001;104(4):531-43.

20. Prevoršek Z, Gorjanc G, Paigen B, Horvat S. Congenic and bionformatics analyses resolved a major-effect Fob3b QTL on mouse Chr 15 into two closely linked loci. Mamm Genome. 2010;21(3-4):172-85.

21. Laplante M, Horvat S, Festuccia WT, Birsoy K, Prevorsek Z, Efeyan A, Sabatini DM. DEPTOR cell-autonomously promotes adipogenesis, and its expression is associated with obesity. Cell Metab. 2012;16(2): 202-12.

22. Morton NM, Beltram J, Carter R, Gorjanc G, Munger SC, Svenson KL, Rodriguez-Cuenca S, Moreno-Navarrete JM, Gibbins M, McFadden C, Gastaldello A, Stott H, Naredo G, Zeyda M, Wang Z, Howie AF, Saari A, Sipila P, Stulnig T, Gudnasson V, Kenyon CJ, Seckl JR, Walker BR, Webster SP, Dunbar DR, Vidal-Puig A, Churchill GA, Fernandez-Real JM, Emilsson V, Horvat S. Genetic selection for extreme low adiposity identifies a healthy leanness gene. Nat Med. 2016. in press.

23. Dreos R, Ambrosini G, Périer R, Bucher P. EPD and EPDnew, high-quality promoter resources in the next-generation sequencing era. Nucleic Acids Res. 2013;41(Database issue):D157-64. http://epd.vital-it.ch/. Accessed 28 May 2015.

24. AliBaba2.1. BIOBASE Biological Databases, Walthman. 2000. http://www.generegulation.com/pub/programs/alibaba2/index.html. Accessed 16 Mar 2015.

25. Daily K, Patel VR, Rigor P, Xie X, Baldi P. MotifMap: integrative genome-wide maps of regulatory motif sites for model species. BMC Bioinformatics. 2011; 12:495. http://motifmap.ics.uci.edu/. Accessed 16 Mar 2015.

26. Kent WJ, Sugnet CW, Furey TS, Roskin KM, Pringle TH, Zahler AM, Haussler D. The human genome browser at UCSC. Genome Res. 2002;12(6):9961006. http://epigenome.usc.edu/resources/software_tools/cpg_island.html. Accessed 4 Dec 2014

27. Li LC, Dahiya R. MethPrimer: designing primers for methylation PCRs. Bioinformatics. 2002;18(11):1427-31. http://www.urogene.org/cgi-bin/ methprimer/methprimer.cgi. Accessed 4 Dec 2014.

28. Takai D, Jones PA. The CpG Island Searcher: A New WWW Resource. In Silico Biol. 2003;3(3):235-40. http://epigenome.usc.edu/resources/software_tools/ cpg_island.html. Accessed 4 Dec 2014.

29. Rice P, Longden I, Bleasby A. EMBOSS: the European Molecular Biology Open Software Suite. Trends Genet. 2000;16(6):276-7. http://www.ebi.ac.uk/ Tools/seqstats/emboss_cpgplot/. Accessed 4 Dec 2014.

30. Gardiner-Garden M, Frommer M. Cpg islands in vertebrate genomes. J Mol Biol. 1987;196(2):261-82.

31. Dweep H, Sticht C, Pandey P, Gretz N. miRWalk - database: prediction of possible miRNA binding sites by "walking" the genes of 3 genomes. J Biomed Inform. 2011;44(5):839-7. http://www.umm.uni-heidelberg.de/ apps/zmf/mirwalk/. Accessed 5 Apr 2015. 
32. Wang X, El Naqa IM. Prediction of both conserved and nonconserved microRNA targets in animals. Bioinformatics. 2008;24(3):325-32. http://mirdb. org/miRDB/. Accessed 5 Apr 2015.

33. MicroCosm Targets Version 5. The European Bioinformatics Institute (EMBL-EBI), Cambridge. http://www.ebi.ac.uk/enright-srv/microcosm/htdocs/ targets/v5/. Accessed 5 Apr.2015.

34. Xiao F, Zuo Z, Cai G, Kang S, Gao X, Li T. miRecords: an integrated resource for microRNA-target interactions. Nucleic Acids Res. 2009;37(Database issue): D105-10. http://c1.accurascience.com/miRecords/. Accessed 5 Apr 2015.

35. Tamura K, Stecher G, Peterson D, Filipski A, Kumar S. MEGA6: Molecular Evolutionary Genetics Analysis Version 6.0. Mol Biol Evol. 2013;30(12):2725-29.

36. Nikolskiy I, Conrad DF, Chun S, Fay JC, Cheverud JM, Lawson HA. Using wholegenome sequences of the LG/J and SM/J inbred mouse strains to prioritize quantitative trait genes and nucleotides. BMC Genomics. 2015;16:415.

37. McCauley JL, Kenealy SJ, Margulies EH, Schnetz-Boutaud N, Gregory SG, Hauser SL, Oksenberg JR, Pericak-Vance MA, Haines JL, Mortlock DP. SNPs in Multi-species Conserved Sequences (MCS) as useful markers in association studies: a practical approach. BMC Genomics. 2007;8:266.

38. Hare MP, Palumbi SR. High intron sequence conservation across three mammalian orders suggests functional constraints. Mol Biol Evol. 2003; 20(6):969-78.

39. Cockerill PN. Structure and function of active chromatin and DNase I hypersensitive sites. FEBS J. 2011;278(13):2182-210.

40. Boyle AP, Davis S, Shulha HP, Meltzer P, Margulies EH, Weng Z, Furey TS, Crawford GE. High-resolution mapping and characterization of open chromatin across the genome. Cell. 2008;132(2):311-22.

41. Southwood CM, Lipovich L, Gow A. Tissue-restricted transcription from a conserved intragenic CpG island in the Klf1 gene in mice. Biol Reprod. 2012;87(5):108.

42. Prieto-Hontoria $P L$, Pérez-Matute $P$, Fernández-Galilea M, Martínez JA, Moreno-Aliaga MJ. Lipoic acid inhibits leptin secretion and Sp1 activity in adipocytes. Mol Nutr Food Res. 2011;55(7):1059-69.

43. Xu H, Uno JK, Inouye M, Collins JF, Ghishan FK. NF1 transcriptional factor(s) is required for basal promoter activation of the human intestinal NaPi-Ilb cotransporter gene. Am J Physiol Gastrointest Liver Physiol. 2005;288(2):G175-81.

44. Lee YH, Sauer B, Johnson PF, Gonzalez FJ. Disruption of the c/ebp alpha gene in adult mouse liver. Mol Cell Biol. 1997;17(10):6014-22.

45. Wang $Y$, Li X, Hu H. H3K4me2 reliably defines transcription factor binding regions in different cells. Genomics. 2014;103(2-3):222-8.

46. Hon GC, Hawkins RD, Ren B. Predictive chromatin signatures in the mammalian genome. Hum Mol Genet. 2009;18(R2):R195-201.

47. Kowalczyk MS, Hughes JR, Garrick D, Lynch MD, Sharpe JA, Sloane-Stanley JA, McGowan SJ, De Gobbi M, Hosseini M, Vernimmen D, Brown JM, Gray NE, Collavin L, Gibbons RJ, Flint J, Taylor S, Buckle VJ, Milne TA, Wood WG, Higgs DR. Intragenic enhancers act as alternative promoters. Mol Cell. 2012; 45(4):447-58.

48. Ivanova N, Dobrin R, Lu R, Kotenko I, Levorse J, DeCoste C, Schafer X, Lun Y, Lemischka IR. Dissecting self-renewal in stem cells with RNA interference. Nature. 2006;442(7102):533-8

49. Loh YH, Wu Q, Chew JL, Vega VB, Zhang W, Chen X, Bourque G, George J, Leong B, Liu J, Wong KY, Sung KW, Lee CW, Zhao XD, Chiu KP, Lipovich L, Kuznetsov VA, Robson P, Stanton LW, Wei CL, Ruan Y, Lim B, Ng HH. The Oct4 and Nanog transcription network regulates pluripotency in mouse embryonic stem cells. Nat Genet. 2006:38(4):431-40.

50. Galan-Caridad JM, Harel S, Arenzana TL, Hou ZE, Doetsch FK, Mirny LA, Reizis B. Zfx controls the self-renewal of embryonic and hematopoietic stem cells. Cell. 2007;129(2):345-57.

51. Li H, Chen D, Zhang J. Analysis of intron sequence features associated with transcriptional regulation in human genes. PLoS One. 2012;7(10):e46784.

52. Wu C, Orozco C, Boyer J, Leglise M, Goodale J, Batalov S, Hodge CL, Haase J, Janes J, Huss JW 3rd, Su Al. BioGPS: an extensible and customizable portal for querying and organizing gene annotation resources. Genome Biol. 2009; 10(11):R130.

53. Barrett LW, Fletcher S, Wilton SD. Regulation of eukaryotic gene expression by the untranslated gene regions and other non-coding elements. Cell Mol Life Sci. 2012;69(21):3613-634.

54. Zhou P, Xu W, Peng X, Luo Z, Xing Q, Chen X, Hou C, Liang W, Zhou J, Wu $X$, Songyang $Z$, Jiang S. Large-scale screens of miRNA-mRNA interactions unveiled that the $3^{\prime} U T R$ of a gene is targeted by multiple miRNAs. PLoS One. 2013;8(7):e68204.
55. Grimson A, Farh KK, Johnston WK, Garrett-Engele P, Lim LP, Bartel DP. MicroRNA targeting specificity in mammals: determinants beyond seed pairing. Mol Cell. 2007;27(1):91-105.

56. Bartel DP. MicroRNAs: target recognition and regulatory functions. Cell. 2009;136:215-33.

57. Keane TM, Goodstadt L, Danecek P, White MA, Wong K, Yalcin B, Heger A, Agam A, Slater G, Goodson M, Furlotte NA, Eskin E, Nellåker C, Whitley H, Cleak J, Janowitz D, Hernandez-Pliego P, Edwards A, Belgard TG, Oliver PL, McIntyre RE, Bhomra A, Nicod J, Gan X, Yuan W, van der Weyden L, Steward CA, Bala S, Stalker J, Mott R, Durbin R, Jackson IJ, Czechanski A, Guerra-Assunção JA, Donahue LR, Reinholdt LG, Payseur BA, Ponting CP, Birney E, Flint J, Adams DJ. Mouse genomic variation and its effect on phenotypes and gene regulation. Nature. 2011;477(7364):289-94.

58. Yang $H$, Wang JR, Didion JP, Buus RJ, Bell TA, Welsh CE, Bonhomme F, Yu AH, Nachman MW, Pialek J, Tucker P, Boursot P, McMillan L, Churchill GA, de Villena FP. Subspecific origin and haplotype diversity in the laboratory mouse. Nat Genet. 2011;43(7):648-55.

59. Didion JP, de Villena FP. Deconstructing Mus gemischus: advances in understanding ancestry, structure, and variation in the genome of the laboratory mouse. Mamm Genome. 2013;24(1-2):1-20.

60. Zorc M, Skok DJ, Godnic I, Calin GA, Horvat S, Jiang Z, Dove P, Kunej T. Catalog of microRNA seed polymorphisms in vertebrates. PLoS One. 2012;7(1):e30737.

61. Morton NM, Nelson YB, Michailidou Z, Di Rollo EM, Ramage L, Hadoke PWF, Seckl JR, Bunger L, Horvat S, Kenyon CJ, Dunbar DR. A stratified transcriptomics analysis of polygenic fat and lean mouse adipose tissues identifies novel candidate obesity genes. PLoS One. 2011;6(9):e23944.

\section{Submit your next manuscript to BioMed Central and we will help you at every step:}

- We accept pre-submission inquiries

- Our selector tool helps you to find the most relevant journal

- We provide round the clock customer support

- Convenient online submission

- Thorough peer review

- Inclusion in PubMed and all major indexing services

- Maximum visibility for your research

Submit your manuscript at www.biomedcentral.com/submit
Biomed Central 